\title{
USO ETNOBOTÂNICO DO MANDACARU (CEREUS JAMACARU DE. CANDOLLE) X BIOLOGIA DA CONSERVAÇÃO: PERCEPÇÃO AMBIENTAL DOS MORADORES DE UMA COMUNIDẢDE RURAL DO SERTÃO PERNAMBUCANO
}

\section{ETHNOBOTANICAL USE OF MANDACARU (CEREUS JAMACARU DE CANDOLLE) $\mathrm{X}$ CONSERVATION BIOLOGY: ENVIRONMENTAL PERCEPTION OF THE DWELLERS OF A RURAL COMMUNITY OF PERNAMBUCO'S SERTÃO}

\author{
Maria Carliana do Nascimento Silva ${ }^{1}$; Francisco Welde Araujo Rodrigues ${ }^{2}$; Dan Vítor Vieira \\ Braga $^{3}$
}

DOI: https://doi.org/10.31692/978-65-991061-7-0.223-231

\section{RESUMO}

A flora do Bioma Caatinga caracteriza-se pela resistência e mudança de aspectos físicos e morfológicos em resposta ao estresse hídrico, sendo isto um dos principais fatores seletivos para as espécies que ocorrem no Bioma A etnobotânica está inclusa em fatores influentes na estrutura e diversidade da vegetação uma vez que a mesma pertence à área da etnobiologia que consiste em uma ciência que estuda as relações do ser humano com as plantas. O presente estudo foi desenvolvido no município de Serrita no Sertão Pernambucano, tendo o povoado São Francisco do Brígida como comunidade foco de estudo. Na localidade, grande parte dos moradores são agricultores tendo então renda baseada na agropecuária. Utilizou-se de método indutivo através de uma pesquisa qualitativa na comunidade a fim da aquisição de dados, foi aplicado questionário semiestruturado com 10 produtores contendo 7 perguntas. A presente pesquisa teve por objetivo descrever as formas de uso, cultivo e status de conservação do Mandacaru em uma comunidade rural frente a exploração agrícola. Todos os entrevistados afirmaram utilizar o Mandacaru como fonte de renda, no entanto a maioria dos entrevistados $(60 \%)$ afirmou fazer uso da planta com uma frequência "média," sendo que a parte da planta mais utilizada são os galhos, argumento afirmado por todos os entrevistados, contudo o método utilizado para explorar a planta concentra-se na extração parcial da mesma. Devido o período de seca prolongada se faz necessário uma maior frequência de uso o que para os produtores as más práticas de exploração deste recurso natural aliado a falta de manejo correto acarretam em danos na planta levando perca da espécime. Portanto o mandacaru é uma fonte de forragem comumente utilizada para a alimentação animal no período de seca e como fonte de renda. Embora necessite de educação ambiental voltando à difusão de formas adequadas de manejo, bem como, de estratégias de recuperação das populações locais de Mandacaru.

Palavras-Chave: Agricultura, Caatinga, Cultivo, Espécie, Educação Ambiental.

\begin{abstract}
\footnotetext{
${ }^{1}$ Licenciatura em Ciências Biológicas, FACHUSC, carliananascimento@outlook.com

${ }^{2}$ Licenciatura em Ciências Biológicas, FACHUSC, franciscoweldearaujo@ outlook.com

${ }^{3}$ Mestre em Gestão e Politicas Ambientais, FACHUSC, bragadvv@gmail.com
}

The flora of the Caatinga Biome is characterized by the resistance and change of physical and morphological aspects in response to water stress, being this one of the main selective factors for the species that occur in the Biome Ethnobotany is included in factors influencing the structure and diversity of the vegetation since it belongs to the area of ethnobiology that consists of a science that studies the relations of the human being with the plants. The present study was developed in the municipality of Serrita in Sertão Pernambucano, with the town of São Francisco do Brígida being the focus of study community. In the locality, a large part of 
the residents are farmers having then income based on agriculture. An inductive method was used through a qualitative research in the community in order to acquire data, a semistructured questionnaire was applied with 10 producers containing 7 questions. The present research had the objective of describing the forms of use, cultivation and conservation status of Mandacaru in a rural community in front of the farm. All respondents stated that Mandacaru was used as a source of income, but most of the respondents $(60 \%)$ stated that they used the plant with a "medium" frequency, and that the most commonly used part of the plant is the branches, an argument affirmed by all However, the method used to explore the plant focuses on the partial extraction of the plant. Due to the prolonged drought period, a greater frequency of use is necessary, which for farmers the bad practices of exploitation of this natural resource, together with the lack of correct management, lead to damages in the plant, leading to loss of the specimen. Therefore mandacaru is a source of forage commonly used for animal feeding in the dry season and as a source of income. Although it needs environmental education, it returns to the diffusion of appropriate forms of management, as well as, strategies of recovery of the local populations of Mandacaru.

Keywords: Agriculture, Caatinga, Cultivation, Species, Environmental education

\section{INTRODUÇÃO}

A Caatinga é o único bioma endêmico ao Brasil, ocupando uma área de cerca de $800.000 \mathrm{~km}^{2}$ que inclui nove estados (Ceará, Rio Grande do Norte, Paraíba, Piauí, Bahia, Alagoas, Sergipe, Pernambuco e Minas Gerais), correspondendo a cerca de $10 \%$ do território nacional. A maior parte de sua área de abrangência geográfica possui clima semiárido, com chuvas escassas e irregulares (PRADO, 2003).

A etnobotânica está inclusa na área da etnobiologia e consiste em uma ciência que estuda as relações do ser humano com as plantas, buscando cada vez mais saber como estas plantas são utilizadas pelas populações tradicionais (ALENCAR, et al. 2015). A questão de sustentabilidade para o uso de recursos florestais é fundamental, quando o assunto é desenvolvimento responsável de uma região, ou comunidade (GARIGLIO, et al. 2010).

O uso de várias espécies da Caatinga é bem conhecido pela comunidade acadêmica, tendo como destaque a família Cactaceae por possui espécies amplamente distribuída por todo o Bioma e, entre elas o Mandacaru (Cereus jamacaru DC.). O mandacaru se destaca na cultura do sertanejo pela sua grande versatilidade e rusticidade, sendo assim, é utilizado de várias formas pelas sociedades tradicionais que residem no Bioma Caatinga, sendo este conhecimento absorvido pelos pecuaristas (LUCENA, et al 2012).

Estes mesmos autores relatam que esta espécie tem principal uso a forragem do cladódio, porém também é conhecido o uso como desta espécie como madeira para construções e urnas funerárias, cerca viva, uso do fruto para a alimentação de animais, na culinária regional, entre outras utilidades (GIRALDI; HANAZAKI, 2010).

Entretanto, a exploração efetuada de forma inadequada tem provocado impactos difusos 
e não quantificados podendo causar a extinção local desta espécie. A presente pesquisa teve por objetivo descrever as formas de uso, cultivo e status de conservação do Mandacaru em uma comunidade rural do sertão central pernambucano.

\section{FUNDAMENTAÇÃO TEÓRICA}

A flora do Bioma Caatinga caracteriza-se pela resistência e mudança de aspectos físicos e morfológicos em resposta ao estresse hídrico, sendo isto um dos principais fatores seletivos para as espécies que ocorrem no Bioma. De acordo com Rodrigues et al (2017), este fator acarretou numa evolução direcional que resultou em um elevado nível de endemismo na vegetação presente no Bioma. O mandacaru (Família: Cactaceae) é uma espécie nativa da vegetação da Caatinga, caracterizado-se por se desenvolver em solo pedregoso sendo encontrado em todos os estados dentro da área de abrangência do Bioma Caatinga (SILVA; ALVES, 2009)

Conforme Leal Sales et al (2014), o mandacaru garante a sobrevivência não só dos animais, mas também dos seres humanos, haja vista que a planta possui diversas formas de utilidade. A agricultura itinerante estar em declínio, por causa da sua baixa produtividade e da impossibilidade de competir com a produção de outras regiões do país exigindo assim formas e estratégias diferentes para que venham gerar mais êxitos (SAMPAIO, 2010).

Para Gandolfo e Hanazaki (2011), uma vez que tanto a cultura quanto a paisagem não são estáticas, as condições para produção de conhecimento etnobotânico também são dinâmicas o que pode contribuir tanto para a manutenção da qualidade de vida quanto para a identificação dos grupos culturais que persistem no local.

As espécies da Caatinga, como o Mandacarú, além da pressão seletiva imposta pelos fatores abóticos extremos característicos da Caatinga, têm suas populações locais sofrido com pressão antrópica das comunidades tradicionais que as utilizam principalmente como forragem para a pecuária extensiva. Isto acaba por causar a extinsão local desta espécie e que somado ao fato das unidades de conservações presentes no bioma Caatinga serem relativamente raras e pequenas acaba por ocasionar impactos significativos na conservação de espécies-chaves (SAMPAIO, 2010). As alterações climáticas e o processo de desertificação tornam a exploração de cactos para fins comerciais uma alternativa viável, por possuírem baixo custo de produção, contudo este cenário requer atenção e precaução (EMBRAPA, 2012), devendo ser desenvolvidas técnicas de manejo sustentável para estas espécies. 


\section{METODOLOGIA}

O presente trabalho foi desenvolvido no município de Serrita-PE, tendo o povoado São Francisco do Brígida como comunidade foco de estudo (Figura 01). A área foco do estudo localiza-se a $35,2 \mathrm{~km}$ da sede do município fica distribuído em um perímetro de cinco quilômetros quadrados com uma população de aproximadamente 250 habitantes (CONCEIÇÃO/2017).

Figura 1. Mapa de Localização da área de Estudo. Fonte: Modificado de Mapas Municipais -Wikipédia, 2018; Google Maps, 2018.

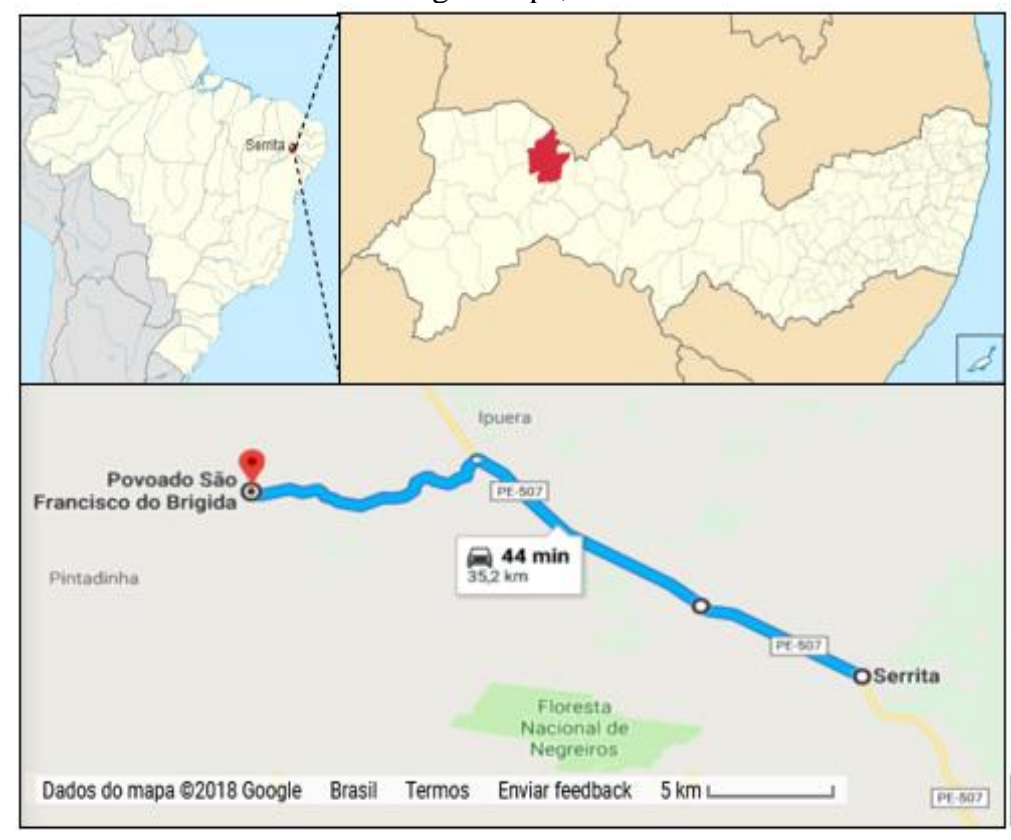

Para obtenção de dados utilizou-se de método indutivo através de uma pesquisa qualitativa na comunidade, haja vista que grande parte dos moradores são agricultores tendo então renda baseada na agropecuária. A região apresenta vegetação de Caatinga ArbustivaArbórea Hiperxerófita e solos raso. A economia local é voltada à agricultura, principalmente nas margens e leito do rio Brígida, sub bacia do rio São Francisco.

O estudo foi realizado no mês de outubro de 2017. A percepção dos produtores residentes na comunidade com relação ao impacto do uso etnobotânico das populações de Mandacaru foi captada através da aplicação de um questionário semiestruturado com 10 produtores com 7 perguntas relacionadas ao tipo de uso, forma de cultivo e percepção quanto a conservação das populações de Mandacaru localizadas nas áreas de entorno da referida comunidade.

\section{RESULTADOS E DISCUSSÃO}

Em relação à exploração do mandacaru todos os entrevistados afirmaram utilizar o 
Mandacaru como fonte de renda, sendo principalmente usado de forma direta através da comercialização (Gráfico 1). Isto corrobora com Sales et al (2014) que nos seus estudos também constataram que o Mandacaru possui grande importância econômica e ambiental para as comunidades rurais, sendo muitas vezes um dos poucos recursos de renda para muitas famílias, e principal recurso alimentar para os animais durante o período de seca prolongada.

Gráfico 1. Utilização da planta como fonte de renda e suas formas de uso. Fonte: NASCIMENTO-SILVA et al. 2018.

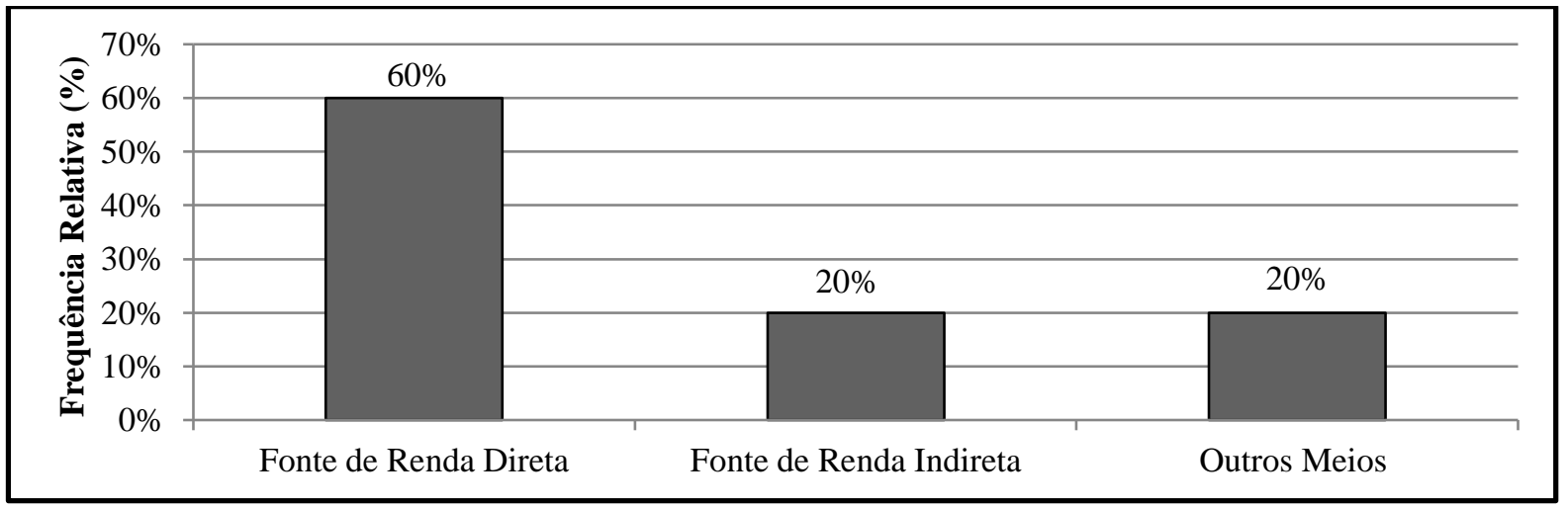

A maioria dos entrevistados (60\%) afirmou fazer uso da planta com uma frequência "média", não acarretando em danos significativos às populações locais de Mandacaru (Gráfico 2). No entanto, isto não corrobora com Santos et al (2017) que afirmam que o mandacaru, geralmente, é usado com maior frequência. De certa forma, esta percepção distorcida da intensidade de exploração das populações de mandacaru pelos produtores é explicado por Rodrigues et al. (2017), onde ressaltam que a população não se incluem como agentes de degradação da vegetação localizada no seu entorno, sendo necessária a execução de trabalhos de educação ambiental com as comunidades, fazendo a explicitação deste nexo causal.

Gráfico 2- Frequência de exploração do Mandacaru na comunidade rural de São Francisco do Brígida, Serrita,

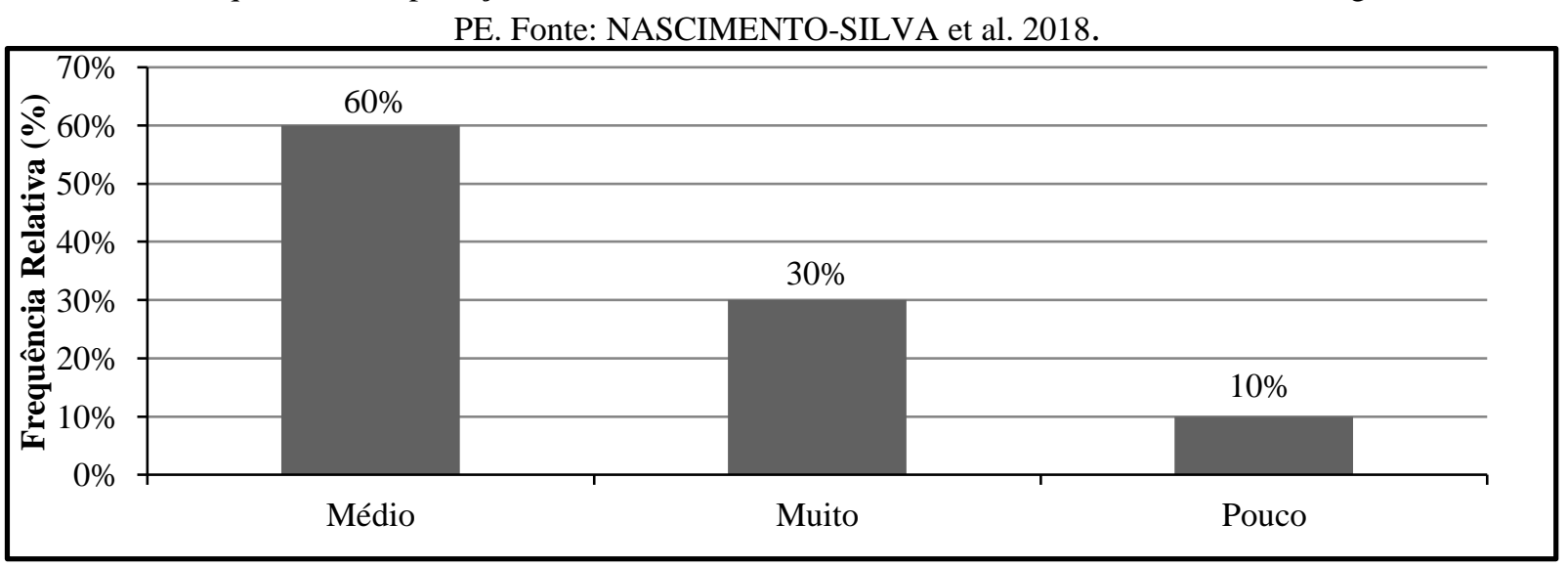

A parte da planta mais utilizada foi os galhos, sendo citado por todos os entrevistados, fator este decorrente da atividade local com maior incidência a agropecuária a qual permite 
manusear da planta para alimentar os animais como o gado, corroborando com Santos, Souza e Amador (2016) que evidenciou este mesmo padrão na comunidade, enfatizando que a visão predominante é que o cacto, em geral, é uma espécie que só é útil como planta forrageira em tempos de secas severas condizendo com a realidade climática da região uma vez que o índice pluviométrico é baixo e concentra-se em poucos meses.

Sobre os métodos utilizados para explorar a planta, 60\% dos produtores afirmaram fazer a extração parcial da mesma, 30\%, extração total e 10\%, argumentaram outros meios não especificados. Para a maioria dos moradores são utilizados apenas os ramos secundários para alimentar os animais (uso forrageiro) e para a retirada de estacas para construção de cercas-vivas. Estas cercas, após estabelecimento dos indivíduos também é utilizada para a retirada de ramos secundários para serem ofertados como forragem aos animais. Lucena et al. (2012) e Sales et al. (2014) que em seus estudos citam, que o Mandacaru é ofertado ao rebanho bovino depois que são espinhos são queimados.

De acordo com $90 \%$ dos entrevistados conhecem cuidados ao explora a planta, sendo que $40 \%$ citaram apenas os galhos, argumentando ser importante para a preservação e recuperação do espécime, porem $40 \%$ afirmaram que é necessária a preservação do caule da planta, para $10 \%$ necessita-se do cultivo sendo exigido regar as mudas, enquanto isso $10 \%$ argumentaram não haver conhecimentos acerca de cuidado ao explorar (gráfico 3). As más práticas de exploração deste recurso natural tornam necessário um olhar atento dos pesquisadores a fim de esclarecer à comunidade da importância do uso de forma sustentável e que isto permitirá a exploração continuada do Mandacaru na área de estudo (SALES ET AL. 2014).

Gráfico 3. Frequência do conhecimento de uso e consequência ao explorar o Mandacaru na comunidade de São Francisco do Brígida Serrita/PE. Fonte: NASCIMENTO-SILVA et al. 2018.

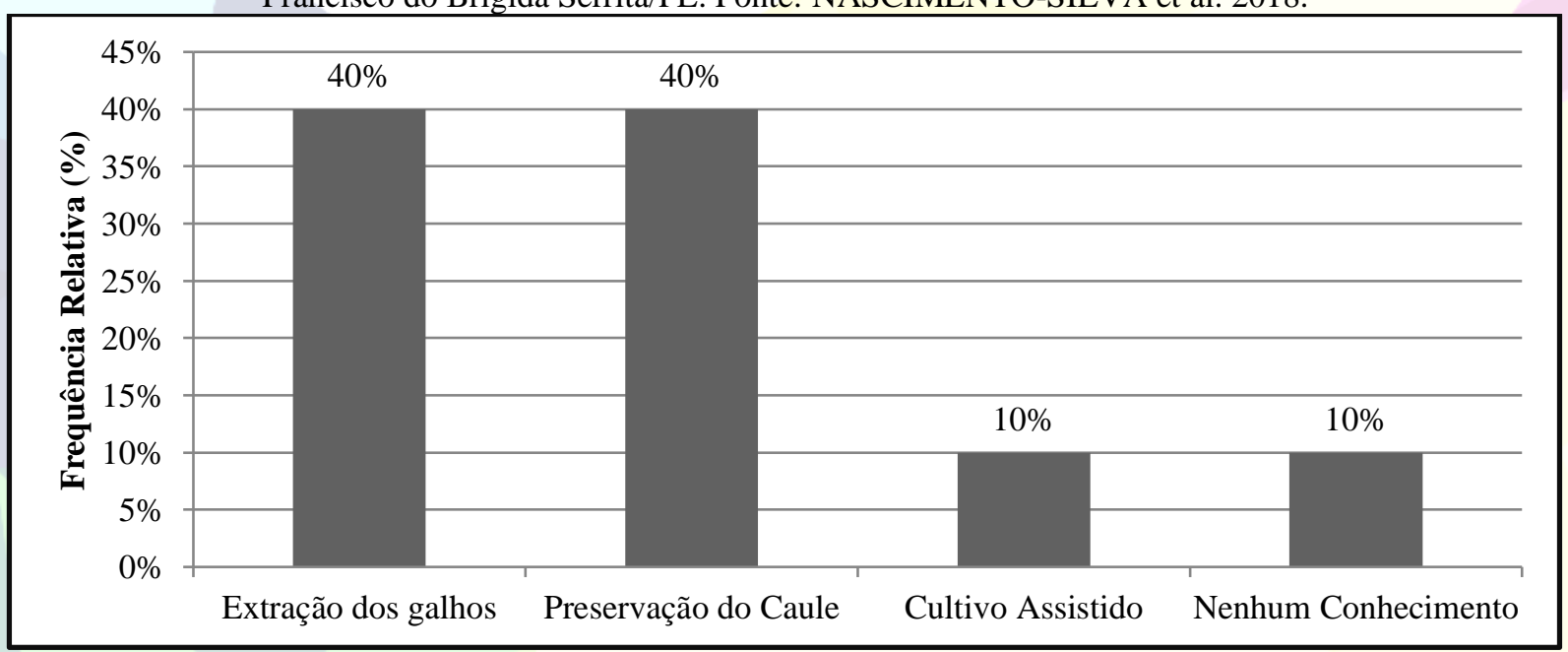


Quanto às consequências do uso inadequado, a maior parte dos informantes $80 \%$ confirmou haver conhecimento, sendo argumentado que pode acarretar na perca da planta, porem foi enfatizado pelos mesmos que a utilização em períodos chuvosos, a planta contém alto teor hídrico, o que após ser aquecida e fornecida para os animais pode provocar a morte dos mesmos, estando de acordo Santana Neto, Castro Filho e Araújo (2015) ao acrescentarem que sendo fornecida como alimento exclusivo, pode causar distúrbios metabólicos nos animais, apresentando queda no desempenho, devido, principalmente, aos baixos teores de constituinte da parede celular.

Outro fator relevante, é que os produtores relataram que no período de seca prolongada se faz necessário uma maior frequência de uso, alterando assim a pressão antrópica sobre as populações de Mandacaru no entorno da comunidade. Este fato também foi mencionado por Albuquerque e Andrade (2002). Segundo estes autores, a manipulação de plantas pelas pessoas em regiões semiáridas depende de vários fatores que vão desde a disponibilidade temporal dos recursos até o grau de interesse por um recurso em especial.

\section{CONCLUSÕES}

O trabalho mostrou que o mandacaru é uma fonte de forragem comumente utilizada pelos produtores da comunidade para a alimentação animal no período de seca e também como fonte de renda. Porém, a forma de exploração adotada causa danos significativos e, muitas vezes, morte dos indivíduos. Como o Mandacaru é uma espécie de recuperação, desenvolvimento e biologia reprodutiva lenta, esta forma de exploração acarreta em diminuição da ocorrência desta espécie nas áreas de entorno da comunidade estudada.

Necessita-se urgentemente da implementação de estratégias de educação ambiental para com os produtores de modo a supri-los de assistência técnica especializada, voltando à difusão de formas adequadas de manejo, bem como, de estratégias de recuperação das populações locais de Mandacaru.

\section{REFERÊNCIAS}

ALBUQUERQUE, U, P; ANDRADE, L, H, C. Conhecimento botânico tradicional e conservação em uma área de Caatinga no estado de Pernambuco, Nordeste do Brasil. Acta Botânica Brasilica. v. 16, n. 3, p.273-285,2002.

ALENCAR, M, Y, de, A; COST, D, A, da; SOUZA, J, B, de; ALENCAR, M, C, B, de, A; CARMO, E, S. Investigação etnobotânica das plantas medicinais utilizadas para o tratamento de faringoamigdalite no CRAS de Cuité, PB. Revista Verde de Agroecologia e Desenvolvimento Sustentável. Pombal-PB-Brasil. 2015, v.10, n.1 , p.1-8. 
CORREIA, D; SILVA, I, C; NASCIMENTO, E, H, S, do; MORAIS, J, P, S; Produção de Mudas de Mandacaru. Embrapa; Circular Técnica 39, Fortaleza-CE, Outubro - 2012. p. 2-6

CONCEIÇÃO, D.A. Ficha Levantamento de Família para Visitas. Agente Comunitário de Saúde São Francisco do Brgída, Serrita-PE, p1 2016.

GANDOLFO, E, S; HANAZAKI, N. Etnobotânica e urbanização: conhecimento e utilização de plantas de restinga pela comunidade nativa do distrito do Campeche (Florianópolis, SC). Acta Botânica Brasílica v. 25, n. 1, p. 168-177. 2011.

GIRALDI, M.; HANAZAKI, N. Use and traditional knowledge of medicinal plants at Sertão do Ribeirão, Florianópolis, Santa Catarina State, Brazil. Acta Botânica Brasilica [online]. 2010, vol.24, n.2, pp.395-406.

GARIGLIO, M, A; SAMPAIO, E, V, de, S, B; CESTARO, L, A; KAGEYAMA, P, Y. (Org.) Uso sustentável e conservação dos recursos florestais da Caatinga. Brasília: Serviço Florestal Brasileiro, 2010. 368p.

LUCENA, C, M, de; COSTA, G, G, da, S; CARVALHO, T, K, N; GUERRA; QUIRINO, G, M; LUCENA, R, F, P, de. Uso e Conhecimento de Cactáceas no Município de São Mamede (Paraíba, Nordeste do Brasil). Revista de Biologia e Farmácia. ISSN 1983-4209-Volume Especial-2012, p. 5-14.

LUCENA, C, M, de; COSTA, G, M, da; SOUSA, R, F, de; CARVALHO, T, K, N; MARREIROS, N, de, A; ALVES, C, A, B; PEREIRA, D, D; LUCENA, R, F, P, de. Conhecimento local sobre cactáceas em comunidades rurais na mesorregião do sertão da Paraíba (Nordeste, Brasil). Revista Biotemas, v.25, n. 3, p. 281-291, setembro de 2012.

PRADO, D, E. As Caatingas da América do Sul. IN: Leal, I.; Tabarelli, M.; Silva, J.M. da (Ed.). Ecologia da Caatinga. Recife: Ed. Universitária da UFPE, 2003. p. 3-5.

RODRIGUES, F, W, A; RODRIGUES, L, A; RODRIGUES, C, A; BRAGA. D, V, V. Uso etnobotânico e conservação de espécies nativas do bioma caatinga: como esta relação é percebida por uma comunidade rural do semiárido pernambucano. Anais. II Congresso Internacional da Diversidade do Semiárido. CAMPINA GRANDE, PB. 2017.

SANTANA-NETO, J, A; CASTRO-FIlHO, E, S; ARAÚJO, H, R, de. Potencial das cactáceas como alternativa alimentar para ruminantes no semiárido Mandacaru, ovinos, palma forrageira. Nutritime Revista Eletrônica, on-line, Viçosa, v.12, n.6, p.4426-4434, nov/dez, 2015. ISSN: 1983-9006 4427.

SALES, M, de, S; MARTINS, V, do L; SOUA, I, de; MEIRELES DE DEUS, M, do, S; PERON, A, P. Cereus jamacaru De Condolle (Cactaceae): o Mandacaru do nordeste brasileiro. Cie. Bio. Saúde, Ponta Grossa, v.20, n.2, p.135-142. jul./dez.2014.

SAMPAIO, E, V, de, S, B. (Org.). Uso sustentável e conservação dos recursos florestais da Caatinga. Brasília: Serviço Florestal Brasileiro, 2010. 368p.: il. Color. ;23cm. 
SANTOS, H, T, dos; SOUZA, M, G, R, de; AMADOR, M, B, M. A percepção do mandacaru (cereus jamacaru) pela população local na paisagem rural de jupi- pe. Revista Homem, Espaço e Tempo março de 2016 ISSN 1982-3800.

SANTOS, J, C, F; SANTOS, J, S, L, do; SILVA, M, R, de; OLEITE, T, da, S. Medidas de conhecimentos, tipos de coleta e uso do Cereus jamacaru (mandacaru) no povoado Quando. Diversista Journal. Santana do Ipanema/AL. vol 2, n.2, p.251-255, mai/ago. 2017.

SILVA, L, R, da; ALVES, R, E. Avaliação da composição físico-química de frutos de mandacaru (Cereus jamacaru P.) Universidade Rural do Semiárido, UFERSA, Mossoró RN, Brasil. Rec. 20. 10. 2009. 